Pneumologe 2020 $\cdot 17: 398-405$

https://doi.org/10.1007/s10405-020-00347-0

Online publiziert: 8. Oktober 2020

(c) Der/die Autor(en) 2020

\section{Redaktion}

H. Olschewski, Graz/Österreich

Für kritisch kranke COVID-19-Patienten könnte das Überleben der Akutphase evtl. nur die Bewältigung der ersten Etappe eines insgesamt langen und herausfordernden Weges sein. Körperliche, kognitive und psychologische Folgen sind realistisch, zumal dies bei SARS-1 und auch ARDS (,,acute respiratory distress syndrome ") in anderem Zusammenhang wiederholt beschrieben wurde. Gerade schwere Verläufe mit komplizierten Intensivaufenthalten und längeren Zeiten mechanischer Beatmung sind ein plausibler Risikofaktor für Folgeerscheinungen bzw. persistierende Symptome. Aber stellen residuale Symptome bei Patienten mit mikrobiologischer Normalisierung tatsächlich ein „Post-COVIDSyndrom" dar?

Unter dem aus dem Griechischen kommenden Begriff „Syndrom“ verstehen wir in der Medizin das Zusammentreffen bzw. gemeinsame Bestehen mehrerer Symptome. Dabei stellt der primär deskriptive Begriff eine gemeinsame Pathogenese der zugleich vorhandenen Symptome in den Vordergrund. Ein „Post-COVID-Syndrom“ würde also 2 Umstände bedingen:

- einerseits das weitere Bestehen mehrerer Symptome nach Abklingen der akuten Krankheitsphase und

- andererseits deren gemeinsame Ursache in der ursprünglichen SARSCoV-2-Infektion.

Hier gilt es vorerst, folgende Frage zu beantworten: Welche Symptome sind in diesem Zusammenhang prinzipiell denkbar und in der Lage, ein Post-COVID-

Bernd Lamprecht

Klinik für Lungenheilkunde, Kepler Universitätsklinikum GmbH, Linz, Österreich

\title{
Gibt es ein Post-COVID-Syndrom?
}

Syndrom zu begründen? Die Persistenz von Fatigue, Dyspnoe und neuropsychologischen Symptomen wird in Abhängigkeit der jeweiligen Studienpopulation sehr häufig berichtet, in $35 \%$ bei ambulant behandelten COVID-Patienten [1] und in $87 \%$ bei Hospitalisierten [2].

Dass kritisch kranke Patienten oftmals über einen längeren Zeitraum nach ihrer Krankenhausentlassung noch funktionelle Einschränkungen erleben, in vielen Fällen sogar über die Dauer von mehreren Jahren, ist nicht neu [3]. Vielen aktuellen Beschreibungen und Analysen von Residualzuständen fehlen jedoch die notwendigen Berücksichtigungen bzw. Adjustierungen für den gesundheitlichen Zustand vor der Infektion mit dem Coronavirus. Genau das aber wäre notwendig, um über ein mögliches Post-COVID-Syndrom belastbare Aussagen machen zu können. Jedenfalls wäre es erforderlich, eine klare Abgrenzung zwischen folgenden Umständen vorzunehmen:

1. Symptome bedingt durch eine persistierende chronische Entzündung,

2. Folgen eines Organschadens (akute Lungen- oder Nierenschädigung) und

3. unspezifische Folgen der Hospitalisation und sozialen Isolation (von ernährungsbedingter Anämie bis hin zum Muskelabbau).

Beispielsweise könnten einfache Laboruntersuchungen durchaus beeinflussbare Hintergründe einer Fatigue identifizieren: Anämie, Vitamin-D-Mangel, Hypothyreose, Kortisoldefizit, chronische Nierenerkrankung [4].

\section{Parallelen zu SARS}

Es gibt Erkenntnisse über ein Virus, das dem gegenwärtigen Coronavirus ähnlich ist: SARS-1, Erreger des schweren akuten respiratorischen Syndroms („severe acute respiratory response syndrome" [SARS]). Nach der SARS-Pandemie von 2003 fiel auf, dass einige Menschen auch Monate und Jahre nach der Infektion mit dem Virus noch gesundheitliche Probleme hatten: $60 \%$ von 117 Befragten berichteten in einer Studie aus Toronto, wo es den größten Ausbruch außerhalb Asiens gegeben hatte, dass sie noch $1 \mathrm{Jahr}$ nach der Entlassung aus dem Krankenhaus an Fatigue litten [5]. In einer anderen Untersuchung, für die SARS-Überlebende aus Hongkong 4 Jahre nach der Infektion befragt wurden, gaben $40 \%$ an, noch unter Fatigue zu leiden [6]. Und der Schlaf- und Schmerzforscher Harvey Moldofsky identifizierte im Gespräch mit SARS-Überlebenden, die selbst nach einem Reha-Programm nicht wieder arbeiten konnten, eine Reihe von Symptomen: Neben anhaltender Fatigue handelte es sich dabei um diffuse Muskelschmerzen, Schwäche, Depressionen und nicht erholsamen Schlaf. Die Beschwerden fasste er unter dem Namen chronisches PostSARS-Syndrom zusammen [7].

\section{Fatigue}

Diese Beschwerden ähneln in ihrer Kombination jenen, von denen auch Menschen mit dem chronischen Fatigue-Syndrom (CFS) berichten. Für eine solche Diagnose ist es aber in den meisten Fällen bei COVID-19 jetzt noch zu früh. Dafür müssen die Symptome mindestens 6 Monate anhalten. Aktuell kann man 
Hier steht eine Anzeige.

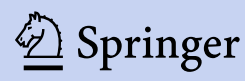


daher wohl nur von postinfektiöser Fatigue sprechen.

\section{》) Aktuell kann man nur von postinfektiöser Fatigue sprechen}

Letztlich ist noch unklar, woher die oftmals geschilderte Abgeschlagenheit kommt. Was aber schon heute sicher ist: Einen einzelnen Grund für die Symptome gibt es nicht. Vielmehr scheinen Veränderungen des Stoffwechsels, des Hormonhaushalts, gegen den eigenen Körper gerichtete Entzündungsbotenstoffe und Veränderungen der Hirnfunktion einen Teil zum Leiden von Fatigue-Patienten beizutragen. Studien deuten etwa darauf hin, dass es den Zellen ihrer Körper schwerer fällt als denen von Gesunden, Energie aus verschiedenen Quellen zu gewinnen [8] - ein wenig so, als würde sich der Körper im Winterschlaf befinden. Eine verminderte Aktivität der Stresshormonachse könnte auch eine gewisse Erschöpfung erklären, denn niedrige Level von Stresshormonen können einerseits dazu führen, dass Entzündungsreaktionen nicht gebremst werden, und andererseits $\mathrm{zu}$ einem niedrigen Blutdruck und Kreislaufbeschwerden.

Auch Entzündungsbotenstoffe spielen womöglich eine Rolle. Bei Menschen, die nach einer Virusinfektion eine chronische Fatigue entwickeln, waren in der Akutphase etwa Interleukin-6 und -10 im Blut stärker erhöht, also exakt die Botenstoffe, die für die überschießende Immunreaktion verantwortlich sind, die COVID-19 so gefährlich macht [9]. Bei postinfektiöser Fatigue sind die entzündlichen Botenstoffe teilweise noch erhöht, obwohl der Mensch schon gesundet ist im Körper könnte also noch immer eine Entzündung schwelen. Dass eine schwelende Entzündung allein die Fatigue erklären könnte, ist jedoch keinesfalls ausreichend belegt. Es wird gegenwärtig die Hypothese formuliert, proinflammatorische Zytokine (Interferon- $\gamma$, Interleukin7) könnten in der postinfektiösen Phase die Blut-Hirn-Schranke passieren und autonome Dysfunktionen verursachen, die sich in einer Dysregulation des SchlafWach-Rhythmus, kognitiver Dysfunkti- on sowie Müdigkeit und Antriebslosigkeit manifestieren können [10].

\section{Dauerhafte Organschäden und mentale Gesundheitsprobleme}

Thromboembolische Komplikationen im Rahmen von COVID-19 wie Pulmonalembolie, Apoplex und andere Mikroinfarzierungen können selbstverständlich eine Vielzahl an dauerhaften Organschäden hervorrufen. Selbst wenn sich Betroffene körperlich wieder erholen, so sind sie evtl. besonders gefährdet, an lang anhaltenden mentalen Gesundheitsproblemen zu leiden. Bei SARS zeigten mehr als ein Drittel der Betroffenen noch 1 Jahr nach körperlicher Erholung eine moderate bis schwere Depression und Ängstlichkeit [11].

\section{Hyperinflammatorische Zustände}

Beobachtungen anderer Rahmenbedingungen, die mit hyperinflammatorischen Zuständen einhergehen, wie SARS, ARDS, Zytokinsturm oder PostICU(,intensive care unit“)-Syndrom, erlauben jedenfalls Spekulationen über mögliche Folgen eines kritischen Verlaufes von COVID-19. Eine Hypothese in diesem Zusammenhang ist, dass ein PostCOVID-Syndrom mit einer chronischen subklinischen systemischen Entzündung (Inflammation) einhergehen könnte, wie dies im Alterungsprozess (Aging) beobachtbar ist. Dieses „Inflammaging“ könnte das Potenzial haben, bestehende Komorbiditäten $\mathrm{zu}$ verschlechtern und altersabhängige Probleme zu verstärken [12].

Bezüglich der Entzündungsreaktion ist inzwischen bekannt, dass eine SARS-CoV-2-Infektion eine starke und oftmals unkontrollierte Entzündungsantwort auslösen kann, die in der Folge zum Gewebeschaden beitragen kann. Diese als „Zytokinsturm“ bezeichnete schwere, systemische Inflammation wird übrigens in allen Altersgruppen beobachtet, bei Kindern wurde eine schwere Multisysteminflammation mit Ähnlichkeiten zum Kawasaki-Syndrom vielfach beschrieben. Hält eine Entzündungsreaktion jedoch über lange Zeit an, so wird angenommen, dass dies $\mathrm{zu}$ zellulärer Seneszenz mit Hemmung der Zellproliferation und Resistenz gegenüber Apoptose führt [12].

\section{Kognitive, psychologische und physische Einschränkungen}

Herridge konnte zeigen, dass Personen nach Erholung von einem ARDS und 5 Jahre nach dem diesbezüglichen Intensivaufenthalt noch immer physische und psychische Limitationen sowie eine reduzierte Lebensqualität empfinden, obwohl sich ihre Lungenfunktionswerte gänzlich oder nahezu vollständig normalisiert hatten [13]. Solche Beobachtungen gibt es jedoch nicht nur nach einem ARDS, viele Intensivpatienten verzeichnen lange anhaltende Beschwerden, die auch als "post-intensive care syndrome“ (PICS) bezeichnet werden. PICS manifestiert sich dabei durch kognitive, psychologische und physische Einschränkungen, die sich in einem relevanten Prozentsatz nicht vollständig zurückbilden [14]. Insofern ist es denkbar, dass eine SARS-CoV-2-Infektion anhaltende Spuren hinterlässt.

\section{Lungenparenchymveränderungen}

Im Rahmen des Ausbruchs von SARS im Jahr 2003 wurden auch längerfristige Auswirkungen wie fibrotische Lungenparenchymveränderungen untersucht. Studienergebnisse zeigten noch 6 Monate nach der Entlassung von Patienten aus der stationären Behandlung eine Einschränkung der Diffusionskapazität bei immerhin $16 \%$ der Betroffenen, und $30 \%$ wiesen zu diesem Zeitpunkt Auffälligkeiten in der Thoraxröntgenaufnahme auf [15]. Bei ARDS (unabhängig von der Ursache) muss bei zumindest $25 \%$ der Überlebenden mit bleibenden Folgen im Sinne einer restriktiven Ventilationsstörung bzw. Lungenerkrankung gerechnet werden [16].

Für die Schwere von bleibenden, in der Bildgebung ersichtlichen bzw. funktionellen Anomalien oder die Wahrscheinlichkeit für Narbengewebe nach ARDS können verschiedene Faktoren eine Rolle spielen. Zu den wichtigsten Einflussfaktoren zählen dabei das Alter des Patienten, vorhandene Komorbiditäten, Rau- 
Hier steht eine Anzeige.

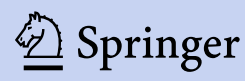


cherstatus, Dauer des Krankenhausaufenthaltes und die Schwere der akuten Erkrankung, beispielsweise die Notwendigkeit einer intensivmedizinischen Versorgung sowie die Art der verabreichten medikamentösen Therapie, also antivirale Medikamente bzw. Kortikosteroide [17].

Radiologisch ersichtliche pulmonale Langzeitfolgen mit Verformung des Lungenparenchyms und konsekutiven Einschränkungen der Lungenfunktion müssen nicht unbedingt der klinischen Symptomatik entsprechen. Zeichen schwerer viraler Pneumonien können in der Bildgebung jedenfalls für einen langen Zeitraum oder sogar dauerhaft ersichtlich bleiben [18]. In diesem Zusammenhang scheint die organisierende Pneumonie in einer signifikanten Anzahl viraler Lungeninfektionen der Ursprung der späteren Veränderungen und Komplikationen zu sein [19]. Bei COVID-19 scheinen die organisierende Pneumonie und der diffuse Alveolarschaden („diffuse alveolar damage" $[\mathrm{DAD}])$, die sich beide in einer weitgehend vorhersehbaren Art und Weise entwickeln, bei Weitem als häufigste Formen von assoziierten Lungenparenchymschäden aufzutreten [20].

\section{》) Es bestehen ausreichend Hinweise für die mögliche Existenz eines Post-COVID- Syndroms}

In Summe bestehen jedenfalls ausreichend Hinweise für die mögliche Existenz eines „Post-COVID-Syndroms“ bzw. für die Berechtigung, die denkbaren Folgeerscheinungen mit persistierenden Symptomen so zu bezeichnen.

\section{Rehabilitationsprogramme}

Günstig für die vollständige und rasche Genesung sowie die Linderung eines Post-COVID-Syndroms kann sich eine gezielte Rehabilitation auswirken. Solche Rehabilitationsprogramme sind dabei sowohl direkt wie auch auf Distanz („remote rehabilitation“) möglich und effektiv [21]. Obwohl diese Rehabilitationsmaßnahmen im Regelfall einen

Pneumologe 2020 - 17:398-405 https://doi.org/10.1007/s10405-020-00347-0

(c) Der/die Autor(en) 2020

\section{B. Lamprecht}

\section{Gibt es ein Post-COVID-Syndrom?}

\section{Zusammenfassung}

Für kritisch kranke COVID-19-Patienten könnte das Überleben der Akutphase evtl. nur die Bewältigung der ersten Etappe eines insgesamt langen und herausfordernden Weges sein. Körperliche, kognitive und psychologische Folgen sind realistisch. Aber stellen residuale Symptome bei Patienten mit mikrobiologischer Normalisierung tatsächlich ein "Post-COVID-Syndrom“ dar, und welche Symptome sind in diesem Zusammenhang prinzipiell denkbar und in der Lage, dieses zu begründen? Dass kritisch kranke Patienten oftmals über einen längeren Zeitraum nach ihrer Krankenhausentlassung noch funktionelle Einschränkungen erleben, ist nicht neu. Für die Diagnose eines PostCOVID-Syndroms ist es aber in den meisten Fällen bei COVID-19 jetzt noch zu früh. Dafür müssen die Symptome mindestens 6 Monate anhalten. Aktuell kann man daher wohl nur von postinfektiöser Fatigue sprechen. Und selbst wenn sich Betroffene körperlich wieder erholen, so sind sie evtl. besonders gefährdet, an lang anhaltenden mentalen Gesundheitsproblemen zu leiden bzw. eine reduzierte Lebensqualität zu empfinden. Solche Beobachtungen gibt es jedoch nicht nur nach einem ARDS (, acute respiratory distress syndrome"), viele Intensivpatienten verzeichnen lange anhaltende Beschwerden, die auch als "post-intensive care syndrome" (PICS) bezeichnet werden. In Summe bestehen jedenfalls ausreichend Hinweise für die mögliche Existenz eines „Post-COVIDSyndroms" bzw. für die Berechtigung, die denkbaren Folgeerscheinungen mit persistierenden Symptomen so zu bezeichnen. Es sind alle Anstrengungen gerechtfertigt, die eine vollständige funktionelle Wiederherstellung und eine Rückkehr in ein Leben nach Corona ermöglichen.

\section{Schlüsselwörter}

Coronavirus · Fatigue · Funktionelle Einschränkungen · Rehabilitation - Folgeerscheinungen

\section{Is there a post-COVID syndrome?}

\section{Abstract}

For critically ill COVID-19 patients surviving the acute phase of the disease could possibly only mean having overcome the first stage of a long and challenging path. Physical, cognitive and psychological consequences seem to be realistic; however, do residual symptoms in patients who have returned to microbiological normalization constitute a post-COVID syndrome and which symptoms are principally possible in this context and are able to cause such a syndrome? It is no novelty that critically ill patients often still sustain functional limitations over a long period after discharge from hospital, in many cases even over many years. In most cases of COVID-19 it is too early for the diagnosis of a post-COVID syndrome. For this the symptoms would have to have lasted over a period of at least 6 months; therefore, only a postinfection fatigue can currently be spoken of. On top of this, even if patients recover physically they could be at particular risk of suffering from long-term mental health problems or perceive a reduced quality of life. Such findings exist not only after ARDS as many intensive care unit patients sustain long-term disorders, which is also known as post-intensive care syndrome (PICS). To sum up, there is sufficient evidence for the possible existence of a post-COVID syndrome or for the justification to correspondingly designate these possible sequelae with persisting symptoms in this way. In any case, all efforts that enable a complete functional recovery and a return to a life after corona are justified.

Keywords

Coronavirus · Fatigue - Functional restrictions . Rehabilitation · Sequelae pneumologischen Schwerpunkt haben, muss gerade auch im Hinblick auf die Vermeidung oder günstige Beeinflussung eines möglichen Post-COVID-Syndroms ebenso auf extrapulmonale (insbesondere neurologische, muskuloskeletale und kardiovaskuläre) Manifestationen und Folgeerscheinungen Bedacht genommen werden [22]. Mit oder ohne erkennbares Post-COVID-Syndrom: Es sind jedenfalls alle Anstrengungen gerechtfertigt, die eine vollständige funktionelle Wie- 
Hier steht eine Anzeige.

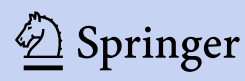


derherstellung und eine Rückkehr in ein Leben nach Corona ermöglichen.

\section{Fazit für die Praxis}

- Bei kritisch kranken COVID-19-Patienten sind körperliche, kognitive und psychologische Folgen der Erkrankung realistisch.

- Für die Diagnose eines Post-COVIDSyndroms ist es in den meisten Fällen bei COVID-19 noch zu früh. Dafür müssen die Symptome mindestens 6 Monate anhalten. Aktuell kann man daher nur von postinfektiöser Fatigue sprechen.

- In Summe bestehen ausreichend Hinweise für die mögliche Existenz eines Post-COVID-Syndroms.

- Um eine vollständige funktionelle Wiederherstellung und eine Rückkehr in ein Leben nach Corona zu ermöglichen, sollten gezielte Rehabilitationsmaßnahmen in Erwägung gezogen werden.

\section{Korrespondenzadresse}

PD Dr. Bernd Lamprecht
Klinik für Lungenheilkunde,
Kepler Universitätsklinikum
GmbH
Krankenhausstr. 9, 4020 Linz,
Österreich
Bernd.lamprecht@kepleruni
klinikum.at

Funding. Open access funding provided by Johannes Kepler University Linz.

\section{Einhaltung ethischer Richtlinien}

Interessenkonflikt. B. Lamprecht gibt an, dass kein Interessenkonflikt besteht.

Für diesen Beitrag wurden vom Autor keine Studien an Menschen oder Tieren durchgeführt. Für die aufgeführten Studien gelten die jeweils dort angegebenen ethischen Richtlinien.

Open Access. Dieser Artikel wird unter der Creative Commons Namensnennung 4.0 International Lizenz veröffentlicht, welche die Nutzung, Vervielfältigung, Bearbeitung, Verbreitung und Wiedergabe in jeglichem Medium und Format erlaubt, sofern Sie den/die ursprünglichen Autor(en) und die Quelle ordnungsgemäß nennen, einen Link zur Creative Commons Lizenz beifügen und angeben, ob Änderungen vorgenommen wurden.
Die in diesem Artikel enthaltenen Bilder und sonstiges Drittmaterial unterliegen ebenfalls der genannten Creative Commons Lizenz, sofern sich aus der Abbildungslegende nichts anderes ergibt. Sofern das betreffende Material nicht unter der genannten Creative Commons Lizenz steht und die betreffende Handlung nicht nach gesetzlichen Vorschriften erlaubt ist, ist für die oben aufgeführten Weiterverwendungen des Materials die Einwilligung des jeweiligen Rechteinhabers einzuholen.

Weitere Details zur Lizenz entnehmen Sie bitte der Lizenzinformation auf http://creativecommons.org/ licenses/by/4.0/deed.de.

\section{Literatur}

1. Tenforde MW (2020) Symptom duration and risk factors for delayed return to usual health among outpatients with COVID-19 in a multistate health care systems network-United States, March-June 2020. MMWR Morb Mortal Wkly Rep 69(30):993-998

2. Carfi A, Bernabei R, Landi F (2020) Persistent symptoms in patients after acute COVID-19. JAMA 324(6):603-605

3. Kress JP, Hall JB (2014) ICU-acquired weakness and recovery from critical illness. N Engl J Med 370(17):1626-1635

4. Garg P, Arora U, Kumar A, Wig N (2020) The "postCOVID" syndrome: how deep is the damage? JMed Virol.https://doi.org/10.1002/jmv.26465

5. Tansey CM, Louie M, Loeb M et al (2007) One-year outcomes and health care utilization in survivors of severe acute respiratory syndrome. Arch Intern Med 167(12):1312-1320.https://doi.org/10.1001/ archinte.167.12.1312

6. Lam MH, Wing YK, Yu MW et al (2009) Mental morbidities and chronic fatigue in severe acute respiratory syndrome survivors: long-term followup. Arch Intern Med 169(22):2142-2147. https:// doi.org/10.1001/archinternmed.2009.384

7. Moldofsky H, Patcai J (2011) Chronic widespread musculoskeletal pain, fatigue, depression and disordered sleep in chronic post-SARS syndrome; a case-controlled study. BMC Neurol 11:37. https:// doi.org/10.1186/1471-2377-11-37

8. Naviaux RK, Naviaux JC, Li Ket al (2017) Metabolic features of chronic fatigue syndrome. Proc Natl Acad Sci U S A 113(37):E5472-E5480. https://doi. org/10.1073/pnas.1607571113

9. Russell A, Hepgul N, Nikkheslat Netal (2019) Persistent fatigue induced by interferon-alpha: a novel, inflammation-based, proxy model of chronic fatigue syndrome. Psychoneuroendocrinology 100:276-285

10. Perrin R, Riste L, Hann M, Walther A, Mukherjee A, Heald A (2020) Into the looking glass: postviral syndrome post COVID-19. Med Hypotheses 144:110055. https://doi.org/10.1016/j.mehy. 2020.110055

11. Lee AM, Wong JG, McAlonan GM et al (2007) Stress and psychological distress among SARS survivors 1 year after the outbreak. Can J Psychiatry 52:233-240

12. Bektas A, Schurman SH, Franceschi C, Ferrucci L (2020) A public health perspective of aging: do hyper-inflammatory syndromes such as COVID-19, SARS, ARDS, cytokine storm syndrome, and postICU syndrome accelerate short- and long-term inflammaging? Immun Ageing 17:23. https://doi org/10.1186/s12979-020-00196-8
13. Herridge MS, Tansey CM, Matte A, Tomlinson G, Diaz-Granados N, Cooper A et al (2011) Functional disability 5 years after acute respiratory distress syndrome. N Engl J Med 364(14):1293-1304

14. Rawal G, Yadav S, Kumar R (2017) Post-intensive care syndrome: an overview. J Transl Int Med 5(2):90-92

15. Hui DS, Joynt GM, Wong KT et al (2005) Impact of severe acute respiratory syndrome (SARS) on pulmonary function, functional capacity and quality of life in a cohort of survivors. Thorax 60(5):401-409. https://doi.org/10.1136/thx.2004. 030205

16. Burnham EL, Hyzy RC, Paine R 3rd et al (2013) Chest CT features are associated with poorer quality of life in acute lung injury survivors. Crit Care Med 41(2):445-456. https://doi.org/10.1097/ CCM.0b013e31826a5062

17. Xie L, Liu Y, Xiao Y, Tian Q, Fan B, Zhao H, Chen W (2005) Follow-up study on pulmonary function and lung radiographic changes in rehabilitating severe acute respiratory syndrome patients after discharge. Chest 127:2119-2124

18. Tang XJ, Chen CC, Tang M, Chen SJ, Zhu ZH, Hou DN, Lu SH, LiT, Bai CX, Song YL (2016) Long-term followup of 5 survivors after the first outbreak of human infections with avian influenza A(H7N9) virus in Shanghai, China. Chin Med J 129:2128-2130

19. Marchiori E, Hochhegger B, Zanetti G (2012) Organising pneumonia as a late abnormality in influenza A (H1N1) virus infection. Br J Radiol 85:841

20. Tian S, Xiong Y, Liu H, Nui L, Guo J, Liao M, Xiao SY (2020) Pathological study oft he 2019 novel coronavirus disease (COVID-19) through postmortem core biopsies. Mod Pathol 33:1007-1014. https:// doi.org/10.1038/s41379-020-0536-x

21. Sakai T, Hoshino C, Yamaguchi R, Hirao $M$, Nakahara R, Okawa A (2020) Remote rehabilitation for patients with COVID-19. J Rehabil Med. https:// doi.org/10.2340/16501977-2731

22. Lopez M, Bell K, Annaswamy T, Juengst S, Ifejika N (2020) COVID-19 guide for the rehabilitation clinician: a review of nonpulmonary manifestations and complications. Am J Phys Med Rehabil 99(8):669-673 
Hier steht eine Anzeige.

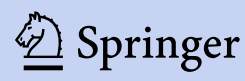

